\title{
Baculovirus-Expressed Nonstructural Protein NS2 of Bluetongue Virus Induces a Cytotoxic T-Cell Response in Mice Which Affords Partial Protection
}

\author{
LINDA D. JONES, ${ }^{1}$ TREVOR WILLIAMS,${ }^{1} \dagger$ DAVID BISHOP ${ }^{1}+$ AND POLLY ROY ${ }^{1,2,3 *}$ \\ Institute of Virology \& Environmental Microbiology, Oxford OX1 $3 S R,{ }^{1}$ and Department of Biochemistry, University of \\ Oxford, Oxford OX1 3QU, ${ }^{2}$ United Kingdom, and Department of International Health, University of Alabama at \\ Birmingham, Birmingham, Alabama $35294^{3}$
}

Received 15 October 1996/Accepted 10 February 1997

\begin{abstract}
Virus-specific cytotoxic T lymphocytes were generated in two strains of mice (BALB/c and CBA/Ca) against baculovirus recombinant proteins (minor and nonstructural) derived from bluetongue virus serotype 10. Immunization of mice with recombinant baculovirus insect cell extracts expressing the nonstructural protein NS2 (Bac-NS2) conferred partial protection against infection with vaccinia virus expressing the NS2 protein. This protective immunity was mediated by $\mathrm{CD8}^{+}$cells. In contrast, no protection was observed when mice were immunized with similarly expressed Bac-NS1 or -NS3 or the virion minor structural proteins (Bac-VP1, -VP4, or-VP6). Furthermore, the in vitro cytotoxicity activity of $T$ cells derived from immunized animals did not correlate to the protective efficacy of baculovirus recombinant proteins. The implications of this work with regard to the design of noninfectious subunit vaccines are discussed.
\end{abstract}

The difficulties associated with obtaining completely safe live-virus vaccines drive the search for new approaches to vaccination. Recombinant DNA technology offers a variety of approaches for the preparation of viral vaccines. With the knowledge of the genetic structures of pathogens, it is possible to assemble, express, and test the utilities of antigenic components which may be required for an effective vaccine. Of virusbased expression vectors, baculoviruses have received considerable attention due to their ability to express large amounts of protein in eukaryotic cells (10). The use of recombinant baculovirus technology in the development of second-generation vaccines is illustrated by the results obtained with bluetongue virus (BTV; genus Orbivirus, family Reoviridae). A vaccine with baculovirus-expressed virus-like particles which mimic authentic BTV virions but lack the RNA (3) has been shown to protect sheep against homologous virus challenge for up to 14 months postinoculation (12). However, in the case of BTV, where several serotypes of the virus can exist within a given area $(4,14)$, a vaccine that provides effective cross-protection is essential (5).

For many virus systems, cytotoxic T lymphocytes (CTLs) which react across serotypic boundaries are elicited to conserved viral proteins, often those that represent the internal components of the virion. Experimental studies have demonstrated the generation of class I-restricted $\mathrm{CD} 8^{+}$CTLs after immunization of two different strains of mice with different BTV serotypes (7). It was observed that the responses to viral internal and nonstructural proteins were predominant, both in the homotypic responses to BTV serotype 10 (BTV-10) and, where observed, in the heterotypic responses. However, the cross-reactivities to individual recombinant proteins in these

\footnotetext{
* Corresponding author. Mailing address: Institute of Virology \& Environmental Microbiology, Mansfield Rd., Oxford OX1 3SR, United Kingdom. Phone: 1865 512361. Fax: 1865 59962. E-mail: por-mail.nerc -oxford.ac.uk.

$\dagger$ Present address: ECOSUR, Tapachula, 30700 Chiapas, Mexico.

$\$$ Present address: St. Cross College, University of Oxford, Oxford OX1 3LZ, United Kingdom.
}

systems were found to be $H$-2 dependent (7). The data supported the concept that certain viral nonstructural proteins are a major source of $\mathrm{T}$-cell antigenic determinants.

Intracellular synthesized antigens are normally processed and associated with class I antigens within cells prior to presentation on the cell surface. In contrast, $\mathrm{CD} 8^{+}$class I-restricted responses which also afford protection have been observed with baculovirus recombinant proteins derived from lymphocytic choriomeningitis virus, vesicular stomatitis virus, and herpesvirus $(1,15)$. Preliminary data have demonstrated that BTV core-like particles synthesized from a dual recombinant baculovirus expressing VP3 and VP7 afforded partial protection in sheep in the absence of neutralizing antibodies (12). However, cell-mediated immune (CMI) responses were not analyzed in those experiments; the mechanism of protection is unresolved.

Here we demonstrate that immunization of mice with either BTV-10 or insect cell extracts from a recombinant baculovirus expressing the NS2 protein of BTV-10 (Bac-NS2) conferred partial protection against vaccinia virus expressing the NS2 protein (VV-NS2). In contrast, recombinant baculoviruses expressing either Bac-NS1 or -NS3 or minor proteins Bac-VP1, -VP4, or -VP6 did not afford protection against challenge with recombinant $\mathrm{VV}$ expressing the homologous protein.

\section{MATERIALS AND METHODS}

Mice. Female BALB/c $\left(H-2^{d}\right)$ and $\mathrm{CBA} / \mathrm{Ca}\left(H-2^{k}\right)$ mice, 8 to 12 weeks old, were obtained from Harlan Olac UK Ltd., Bicester, United Kingdom.

Cell lines and viruses. BHK-21 and Vero cells were grown as monolayers in Eagle's minimal essential medium containing 10\% fetal bovine serum (FBS) at $37^{\circ} \mathrm{C}$ in $5 \% \mathrm{CO}_{2}$. Target P815 $\left(H-2^{d}\right)$ and $\mathrm{L} 929\left(H-2^{k}\right)$ cells were obtained from the Sir William Dunn School of Pathology, University of Oxford, Oxford, United Kingdom. Both cell lines were propagated in RPMI-10 (RPMI 1640 medium containing $10 \%$ FBS) and maintained as described above. Spodoptera frugiperda (Sf21) cells were grown in suspension cultures at $28^{\circ} \mathrm{C}$ in TC100 medium supplemented with $10 \%$ FBS.

The United States prototype isolate of BTV-10 was originally obtained from the Animal Disease Research Laboratory, Denver, Colo. The virus was plaque cloned by using monolayers of BHK-21 cells, and the serotype was verified by a monotypic serum neutralization test (data not shown). Recombinant VVs and recombinant baculoviruses expressing each of the full-length BTV-10 minor structural proteins (VP1, VP4, or VP6) or nonstructural proteins (NS1, NS2, or 
(a)

$H-2^{d}$

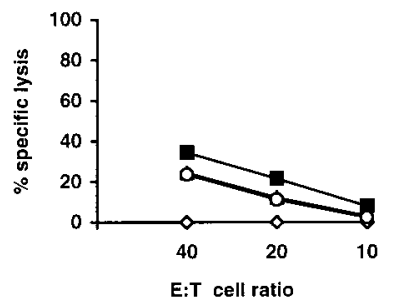

(b)

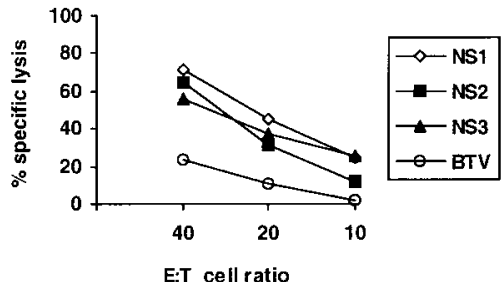

(c)

$H \cdot 2^{k}$

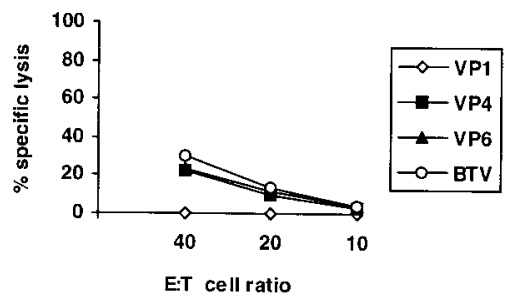

(d)

$H-2^{k}$

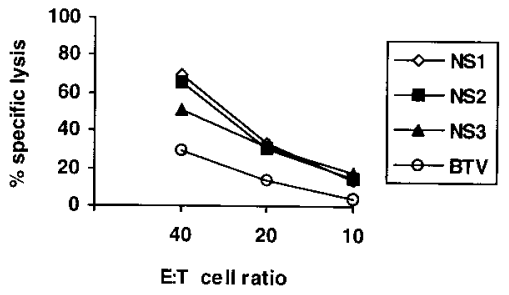

FIG. 1. Lymphocyte effector cells derived from mice inoculated either with Bac-VP1, Bac-VP4, Bac-VP6, or BTV-10 (O) (a and c) or with Bac-NS1, Bac-NS2, Bac-NS3, or BTV-10 $(\mathrm{O})(\mathrm{b}$ and d) were prepared as described in Materials and Methods. Target cells were infected for $24 \mathrm{~h}$ with 50 PFU of BTV-10 per cell prior to incubation with effector cells for $4 \mathrm{~h}$ in a nonradioactive cytotoxicity assay.

NS3) were generated as previously described $(7,13)$. The expression of BTV proteins was confirmed by indirect immunofluorescence antibody tests and Western blot analysis (data not shown).

Preparation of recombinant viral proteins. Spinner cultures $(200 \mathrm{ml})$ of Sf21 cells at a density of $10^{6}$ cells $/ \mathrm{ml}$ were infected with either wild-type Autographa californica nuclear polyhedrosis virus (AcNPV) or recombinant baculoviruses expressing either BTV-10 VP1, VP4, VP6, NS1, NS2, or NS3 at a multiplicity of infection (MOI) of $2 \mathrm{PFU} /$ cell. Cultures were incubated for $56 \mathrm{~h}$ prior to being harvested. Cells were disrupted by sonication, and unpurified extracts were stored at $-20^{\circ} \mathrm{C}$. The presence of antigens was confirmed by Western blot analysis, and the concentrations of expressed proteins were estimated by sodium dodecyl sulfate gel analysis after staining and by comparison to known amounts of bovine serum albumin.

CTL generation and assays. Female $\mathrm{BALB} / \mathrm{c}$ and $\mathrm{CBA} / \mathrm{Ca}$ mice were inoculated intraperitoneally (i.p.) with $7.0 \log _{10}$ PFU of BTV-10 or with cell extracts containing approximately $20 \mu \mathrm{g}$ (each) of the BTV-10-expressed minor and nonstructural proteins. Mice were sacrificed at 7 days postinoculation, and their spleens were removed. Splenocytes from immunized mice were prepared by standard methods (9) and cocultured for 7 days with autologous BTV-10-infected irradiated spleen cells from nonimmunized mice (7). Antigen-presenting cells were infected with BTV at an MOI of $10 \mathrm{PFU} /$ cell and cocultivated with effector cells at a ratio of 1:2. Effector cells (from three individual mice) and antigen-presenting cells were resuspended in RPMI-10 containing $10 \mathrm{mM}$ HEPES, $100 \mathrm{U}$ of penicillin per $\mathrm{ml}, 100 \mu \mathrm{g}$ of streptomycin, $0.03 \%$ glutamine, and $30 \mu \mathrm{M}$ 2-mercaptoethanol and incubated at $37^{\circ} \mathrm{C}$ in $5 \% \mathrm{CO}_{2}$. Target $(\mathrm{P} 815$ and L929) cells were grown to confluency at $37^{\circ} \mathrm{C}$ and infected for $24 \mathrm{~h}$ with BTV-10 at an MOI of 50 PFU/cell or mock infected. Cells were washed twice in RPMI-10 and resuspended at a final concentration of $5 \times 10^{4}$ cells $/ \mathrm{ml}$.

Putative effector cells from each culture were tested in a nonradioactive cytotoxicity assay (Cytotox 96; Promega, Madison, Wis.) as previously described (7). Briefly, this assay is a colorimetric alternative to ${ }^{51} \mathrm{Cr}$ release cytotoxicity assays and quantitatively measures lactate dehydrogenase (LDH), a stable cytosolic enzyme that is released upon cell lysis. The released LDH in culture supernatants was measured by a 30-min coupled-enzyme assay which results in the conversion of a tetrazolium salt of the substrate to a red formazan product.

One hundred microliters of target cells was added to triplicate wells in a 96-well plate to test the following effector/target cell ratios: 40:1, 20:1, and 10:1. Target cell spontaneous release was measured by adding the same concentration of target cells to triplicate sets of wells containing $100 \mu$ l of medium. Cytotoxicity assay plates were centrifuged at $275 \times g$ for $4 \mathrm{~min}$ prior to incubation at $37^{\circ} \mathrm{C}$ in $5 \% \mathrm{CO}_{2}$ for $4 \mathrm{~h}$. Then plates were centrifuged at $275 \times g$ for $4 \mathrm{~min}$, and $50-\mu \mathrm{l}$ aliquots from all wells were transferred to fresh 96-well plates prior to the addition of $50 \mu \mathrm{l}$ of substrate to each well. Plates were incubated for $30 \mathrm{~min}$ at room temperature prior to the addition of $50 \mu$ l of the "stop" solution (Cytotox 96). Absorbance values at $492 \mathrm{~nm}$ were recorded within $1 \mathrm{~h}$ of the addition of the stop solution.

The results were calculated as follows. The average absorbance values from the culture medium background were subtracted from all absorbance values, except for those obtained from the target cell maximum LDH release. In these cases, the average absorbance values from the volume correction controls were subtracted. By using the corrected values, the following formula was used to compute the percent cytotoxicity for each effector/target cell ratio: (experimental - effector spontaneous - target spontaneous)/(target maximum - target spontaneous) $\times 100$.

In all experiments, samples were run in triplicate and showed a variance of less than $8 \%$. The data in Fig. 1 are for three animals per inoculum.

Mouse immunization and challenge experiments. The protective effects mediated by CTLs specific for the BTV minor or nonstructural proteins could not be evaluated by challenging mice with BTV-10, as the virus does not cause disease in adult mice. Thus, the protective efficacies of baculovirus recombinant proteins were assessed by challenging mice with recombinant VVs expressing the homologous proteins and assaying the $\mathrm{VV}$ titers in the ovaries. BALB/c and $\mathrm{CBA} / \mathrm{Ca}$ mice were immunized i.p. with $20 \mu \mathrm{g}$ of either BTV Bac-VP1, -VP4, -VP6, -NS1, -NS2, or -NS3 or BTV (7.0 $\log _{10}$ PFU) as described previously; control mice were immunized with $20 \mu \mathrm{g}$ of AcNPV. Nine days later, mice were challenged i.p. with $6.7 \log _{10}$ PFU of recombinant VV expressing the corresponding protein. Control mice were correspondingly challenged with recombinant VV expressing each of the minor or nonstructural proteins. Mice were sacrificed at 5 days postinoculation, and the recombinant $\mathrm{VV}$ titers in their ovaries were determined (titers were expressed for total ovarian tissue/mouse) (2).

Depletion of CD4 and CD8 T cells. The depletion of T-cell populations in donor mice was undertaken as previously described (1) with monoclonal antibodies YTS 191.1, specific for CD4, and YTS 169.4, specific for CD8, obtained from the Sir William Dunn School of Pathology. To assess the effects of cell depletion on protective immunity, mice were given $1 \mathrm{mg}$ of the appropriate antibody on days 3 and 1 prior to the experiment. After administration of the appropriate antibody (anti-CD4 or anti-CD8), satisfactory depletion of the relevant cell populations was confirmed by fluorescence-activated cell sorting ( $\leq 10,000$ cells) (data not shown).

Statistical analysis. Protection data were analyzed by using a generalized linear statistical model with the logarithm of the ovarian titers as the $y$ variable. A normal error structure was specified, and the treatments were analyzed sequentially as factors. The significance of each treatment was assessed by calculating $F$ ratios from the changes in model deviance (adjusted sums of squares) given by the program. Pairwise comparisons between means were achieved by calculating the standard error (SE) of the differences between means and performing a standard $t$ test.

\section{RESULTS}

Cytotoxic responses of $\mathrm{BALB} / \mathrm{c}$ and $\mathrm{CBA} / \mathrm{Ca}$ mice to recombinant baculoviruses expressing $\mathrm{BTV}-10$ minor and nonstructural proteins. BALB/c $\left(H-2^{d}\right)$ and $\mathrm{CBA} / \mathrm{Ca}\left(H-2^{k}\right)$ mice were immunized with $20 \mu \mathrm{g}$ of either BTV Bac-VP1, -VP4, -VP6, 
TABLE 1. Bac-NS2-primed mice are partially protected from the replication of recombinant VV expressing the homologous protein

\begin{tabular}{|c|c|c|c|c|c|}
\hline \multirow{2}{*}{$\begin{array}{c}\text { Primary } \\
\text { inoculum }^{a}\end{array}$} & \multirow{2}{*}{$\begin{array}{l}\text { Challenge } \\
\text { inoculum }\end{array}$} & \multicolumn{2}{|c|}{$H-2^{k}$ haplotype } & \multicolumn{2}{|c|}{$H-2^{d}$ haplotype } \\
\hline & & $\begin{array}{c}\text { No. of mice } \\
\text { infected/no. tested }\end{array}$ & $\begin{array}{l}\text { Mean titer in ovaries } \\
\left(\log _{10} \mathrm{PFU}\right) \pm \mathrm{SE}^{b}\end{array}$ & $\begin{array}{c}\text { No. of mice } \\
\text { infected/no. tested }\end{array}$ & $\begin{array}{l}\text { Mean titer in ovaries } \\
\left(\log _{10} \text { PFU }\right) \pm \mathrm{SE}^{b}\end{array}$ \\
\hline Bac-VP1 & VV-VP1 & $10 / 10$ & $6.83 \pm 0.30$ & $10 / 10$ & $7.17 \pm 0.30$ \\
\hline BTV-10 & & $10 / 10$ & $7.00 \pm 0.23$ & $10 / 10$ & $7.22 \pm 0.23$ \\
\hline AcNPV & & $10 / 10$ & $7.19 \pm 0.18$ & $10 / 10$ & $7.19 \pm 0.20$ \\
\hline Bac-VP4 & VV-VP4 & $10 / 10$ & $6.93 \pm 0.22$ & $10 / 10$ & $7.26 \pm 0.27$ \\
\hline BTV-10 & & $10 / 10$ & $6.89 \pm 0.29$ & $10 / 10$ & $7.05 \pm 0.23$ \\
\hline AcNPV & & $10 / 10$ & $7.13 \pm 0.33$ & $10 / 10$ & $7.21 \pm 0.16$ \\
\hline Bac-VP6 & VV-VP6 & $8 / 10$ & $6.66 \pm 0.24$ & $10 / 10$ & $6.90 \pm 0.31$ \\
\hline BTV-10 & & $9 / 10$ & $6.54 \pm 0.26$ & $10 / 10$ & $6.85 \pm 0.38$ \\
\hline AcNPV & & $10 / 10$ & $6.73 \pm 0.24$ & $10 / 10$ & $7.17 \pm 0.25$ \\
\hline Bac-NS1 & VV-NS1 & $8 / 10$ & $6.49 \pm 0.41$ & $10 / 10$ & $6.67 \pm 0.24$ \\
\hline BTV-10 & & $10 / 10$ & $5.53 \pm 0.46$ & $10 / 10$ & $5.26 \pm 0.34$ \\
\hline AcNPV & & $10 / 10$ & $7.23 \pm 0.23$ & $10 / 10$ & $7.28 \pm 0.20$ \\
\hline Bac-NS2 & VV-NS2 & $7 / 10$ & $4.15 \pm 0.24$ & $10 / 10$ & $4.20 \pm 0.19$ \\
\hline BTV-10 & & $8 / 10$ & $3.97 \pm 0.28$ & $10 / 10$ & $4.15 \pm 0.14$ \\
\hline AcNPV & & $10 / 10$ & $7.24 \pm 0.24$ & $10 / 10$ & $7.24 \pm 0.23$ \\
\hline Bac-NS3 & VV-NS3 & $10 / 10$ & $7.25 \pm 0.20$ & $10 / 10$ & $7.11 \pm 0.33$ \\
\hline BTV-10 & & 9/10 & $5.12 \pm 0.25$ & $9 / 10$ & $5.29 \pm 0.26$ \\
\hline AcNPV & & $10 / 10$ & $7.32 \pm 0.14$ & $10 / 10$ & $7.17 \pm 0.24$ \\
\hline
\end{tabular}

${ }^{a}$ Mice were immunized with either live BTV-10, recombinant baculoviruses expressing one of the minor or nonstructural proteins, or AcNPV (controls). Nine days later, animals were challenged with recombinant VV expressing the corresponding BTV protein. Ovaries were removed at 5 days postchallenge (see Materials and Methods).

${ }^{b}$ The mean titers \pm SEs were calculated from ovaries found to contain virus.

-NS1, -NS2, or -NS3 protein; control mice were immunized with BTV-10 virus. Lymphocytes derived from immunized mice were assayed for their capacity to lyse in vitro target cells infected with BTV-10 virus (Fig. 1).

Baculovirus recombinants containing individual genes from BTV-10 virus encoding VP4, VP6, NS1, NS2, or NS3 protein elicited BTV-10-specific CTLs in both BALB/c and CBA/Ca mice. In contrast, BTV-10-specific CTLs were not detected among splenic lymphocytes obtained from either strain of mice inoculated with Bac-VP1. Control target cells (mock infected) were not lysed by the effector cell populations ( $0 \%$ lysis). No significant differences in lysis values were observed between the two haplotypes of mice. The nonstructural proteins dominated the CTL responses in both $\mathrm{BALB} / \mathrm{c}$ and $\mathrm{CBA} / \mathrm{Ca}$ mice. The highest levels of cell lysis were observed with effector cells derived from mice immunized with Bac-NS1, with means of $70.0 \%$ (58.9 to $86.0 \%$ ) and $71.6 \%$ (62.3 to $78.2 \%$ ), respectively (parenthetical data are $95 \%$ confidence limits).

Protective immunity. The protective effects of baculovirusexpressed BTV-10 minor and nonstructural proteins were assessed by measuring virus titers in the ovaries of mice challenged with homologous VV recombinants (Table 1). No significant haplotype effect $\left(F_{1,330}=2.03\right.$ [not significant $\{\mathrm{NS}\}]$ ) was observed. The mean $\log _{10} \mathrm{PFU}$ titer $\pm \mathrm{SE}$ in $\mathrm{CBA} / \mathrm{Ca}\left(H-2^{k}\right)$ ovaries was $6.49 \pm 0.12$, compared to $6.55 \pm$ 0.12 in BALB/c $\left(H-2^{d}\right)$ mice. However, the mean proportion of animals which became infected was slightly higher for $H-2^{d}$ haplotype mice, $99.4 \%$ (99.9 to $95.7 \%$ [95\% confidence limits]), compared to that of $H-2^{k}$ mice, $93.9 \%$ (96.6 to $89.2 \%$ ).

Although a marked primary inoculum effect was observed $\left(F_{2,345}=32.1[P<0.001]\right)$, there was no difference in the ovarian titers of the two haplotypes of mice used. Therefore, for pairwise comparisons, the data were pooled. The mean log titer in control mice immunized with AcNPV was $7.18 \pm 0.06$, which was significantly higher than the VV titers observed in the ovaries of mice previously immunized with recombinant baculoviruses expressing BTV-10 minor or nonstructural proteins, $6.53 \pm 0.12(t=4.28$ [df 229, $P<0.05])$. The lowest viral titers were observed in the ovaries of mice immunized with BTV-10, $5.96 \pm 0.13(t=3.7$ [df 224, $P<0.05])$.

When the primary inoculum treatments were considered individually, the majority of treatments were not significantly different from one another. Although the titers obtained from mice immunized with BTV-10 and challenged with VV-NS1 or VV-NS3 protein were not significantly different from one another, they were lower than, for example, either mouse strain challenged with VV-VP1, a typical representative of the other BTV protein treatments $\left(1.60 \log _{10}[t=6.06\right.$, df $38 ; P<0.05]$ and $1.79 \log _{10}[t=7.01$, df $36 ; P<0.05]$, respectively). The titers from mice immunized with either BTV-10 or Bac-NS2 and challenged with VV-NS2 were 2.93 and $2.82 \log _{10}$ lower, respectively, compared to those of control mice immunized with AcNPV. Furthermore, both of these inocula gave VV titers lower than those obtained from mice immunized with BTV-10 and challenged with either VV-NS1 $(t=4.92$ [df 36, $P<0.05$ ] and $t=4.56$ [df 35, $P<0.05$ ], respectively) or VV-NS3 $(t=4.32$ [df 34, $P<0.05$ ] and $t=3.85$ [df 33, $P<$ $0.05]$, respectively).

Although a significant challenge effect was observed $\left(F_{5,347}\right.$ $=16.5[P<0.01])$, animals challenged with VV-VP1, -VP4, or -VP6 did not show differences in ovarian titers. Animals challenged with VV-NS2 had lower titers in their ovaries than with any other treatments. VV-NS1 and VV-NS3 gave higher titers than did VV-NS2 $(t=5.79$ [df 111, $P<0.05$ ] and $t=6.30$ [df $111, P<0.05]$, respectively), but both were significantly lower than those for the VV-expressed minor protein treatments (for $\mathrm{NS} 1, t=3.06$ [df 116, $P<0.05$ ]; for NS3, $t=2.62$ [df $116, P<$ $0.05])$, compared to that of VV-VP1. 
TABLE 2. The depletion of $\mathrm{CD}^{+}$cells in mice abrogates protection against challenge with VV-NS2

\begin{tabular}{ccccc}
\hline \multirow{2}{*}{ Time (days) } & Treatment $^{b}$ & \multicolumn{3}{c}{ Mean titer in ovaries $\left(\log _{10}{\text { PFU }) \pm \text { SE }^{a}}\right.$} \\
\cline { 3 - 5 } & & Bac-NS2 & BTV-10 & Unprimed \\
\hline \multirow{2}{*}{9} & Untreated & $3.72 \pm 0.24(4 / 5)$ & $3.97 \pm 0.16(3 / 5)$ & $7.37 \pm 0.16(5 / 5)$ \\
& Anti-CD8 & $7.20 \pm 0.15(5 / 5)$ & $7.11 \pm 0.21(5 / 5)$ & ND $^{d}$ \\
21 & & $3.71 \pm 0.23(5 / 5)$ & $7.15 \pm 0.33(5 / 5)$ \\
& Untreated & $3.99 \pm 0.26(4 / 5)$ & $3.65 \pm 0.21(4 / 5)$ & ND \\
& Anti-CD4 & $3.74 \pm 0.24(5 / 5)$ & $6.86 \pm 0.25(5 / 5)$ & ND \\
\hline
\end{tabular}

${ }^{a}$ The mean titers \pm SEs were calculated from ovaries found to contain virus. Parenthetical data are ratios of the number of mice infected to the number of mice tested.

${ }^{b}$ Groups of five mice per treatment were used. Ovaries were removed 5 days after mice were challenged with VV-NS2.

${ }^{c}$ Before immunization.

${ }^{d} \mathrm{ND}$, not done.

In summary, these results indicate that the immunization of mice with either Bac-NS2 or BTV-10 and subsequent challenge with VV-NS2 resulted in a significant reduction in the ovarian titers of mice at 5 days postchallenge. Similar results were obtained when mice were immunized with BTV-10 and challenged with either VV-NS1 or VV-NS3. In contrast, no consistent reduction in titer was observed when mice were immunized with Bac-NS1 or Bac-NS3 protein. This indicates that previous immunization with Bac-NS1 or Bac-NS3 did not afford protection against challenge with recombinant VV expressing the homologous protein, even though CTL responses could be demonstrated by in vitro assays. Furthermore, the observation that mice immunized with BTV-10 and challenged with VV-VP1, -VP4, or -VP6 did not show a reduction in ovarian titer indicates that the response observed for Bac-NS2 was specific and was not due to nonspecific suppression of $\mathrm{VV}$ replication.

Effects of in vivo depletion of $\mathrm{CD4}^{+}$or $\mathrm{CD8}^{+} \mathrm{T}$ cells on the immune response. Mice which were either untreated or previously depleted of $\mathrm{CD}^{+}$or $\mathrm{CD}^{+} \mathrm{T}$ cells were immunized with either BTV-10 or Bac-NS2 or unprimed, and 9 or 21 days later, all mice were challenged with VV-NS2 (Table 2). Overall, the $\log$ mean titers \pm SEs for $\mathrm{BALB} / \mathrm{c}$ and $\mathrm{CBA} / \mathrm{Ca}$ mice were $5.62 \pm 0.32$ and $5.62 \pm 0.23$, respectively. No significant difference in ovarian titers was observed between the two haplotypes of mice $\left(F_{1,103}=0.49[\mathrm{NS}]\right)$, and there was no detectable effect on the observed virus titers in relation to time of challenge $\left(F_{1,94}=0.22[\mathrm{NS}]\right)$. However, a very clear treatment effect was observed in both haplotypes of mice $\left(F_{11,114}=9.34\right.$ $[P<0.01])$. At 9 days, the VV titers in the ovaries of nondepleted mice were not significantly different, although they were clearly lower than those in unprimed mice and anti-CD8-depleted mice. Similar results were obtained when mice were challenged at 21 days postinoculation. The titers in the ovaries of $\mathrm{CD} 4^{+}$-depleted mice were similar to those of nondepleted mice. Thus, when $\mathrm{CD}^{+} \mathrm{T}$ cells were depleted prior to challenge with VV-NS2, the protection was abrogated. Similarly, when mice were depleted of $\mathrm{CD}^{+} \mathrm{T}$ cells before immunization with BTV or Bac-NS2, they were not protected against VV-NS2 challenge. The depletion of $\mathrm{CD}^{+}{ }^{+} \mathrm{T}$ cells did not abrogate protection against VV-NS2.

\section{DISCUSSION}

In this study, we demonstrated that noninfectious BTV viral antigens stimulated a CTL response in vivo and efficiently recognized target cells in vitro. A single immunization of $\mathrm{BALB} / \mathrm{c}$ or $\mathrm{CBA} / \mathrm{Ca}$ mice with cell extracts derived from recombinant baculoviruses expressing each of the nonstructural proteins (NS1, NS2, and NS3) and two of the three minor proteins, VP4 and VP6, induced primary CTL activity. In accordance with the results obtained from our previous study, we found that of the antigens studied, the nonstructural proteins were the dominant antigens in both haplotypes of mice (7). The observation that Bac-VP1 did not stimulate a primary CTL response may be due to impaired antigen presentation, as polyclonal CTL populations derived from mice immunized with live BTV-10 recognize target cells expressing VP1 (7).

There are several previous examples of baculovirus-derived immunogens providing a protective $\mathrm{CD}^{+}$class I-restricted response $(1,15)$. However, in the case of BTV-10, partial protection was observed only in mice inoculated with BTV-10 and challenged with recombinant VV expressing the nonstructural proteins or inoculated with Bac-NS2 protein and challenged with VV-NS2. No protection was observed when mice were immunized with recombinant baculoviruses expressing any of the minor structural proteins. As CTLs differ in their protective capacity in vivo depending on the epitope(s) recognized (6), it is possible that the epitopes on the minor proteins and Bac-NS1 and -NS3 are not capable of conferring protection in vivo in this system. Furthermore, in this study, resistance to subsequent virus challenge did not correlate with all the primary CTL activities.

The observation that primary CTL induction does not always confer antiviral protection has also been made with influenza virus (8). Mice immunized with the $K^{d}$-restricted influenza virus nucleoprotein displayed primary nucleoproteinCTL activity but were not resistant to influenza virus respiratory infection in vivo. Although the immunological mechanisms which underlie these phenomena are undetermined, those researchers postulated that one or more of the following situations could contribute to these results. (i) Insufficient numbers of CTLs are present at the site of virus challenge. (ii) $\mathrm{CD}^{+} \mathrm{T}$ cells with high densities and/or affinities for T-cell-specific epitopes might be selectively amplified in vitro, or alternatively in vitro stimulation optimizes the secretion of antiviral molecules. (iii) The presence of T-helper cells specific for the virus may augment resistance mediated by CTLs induced by immunization in vivo. However, for BTV, there is evidence that CMI response plays a role in protection. Thus, it can be concluded that in some virus systems, the presence of in vitro cytotoxicity of primary CTLs derived from vaccinated animals is not a good indicator of in vivo resistance to virus challenge.

The serotype-specific BTV VP2 protein is considered to be the main viral antigen responsible for inducing neutralizing responses in vaccinated animals. However, preliminary crossprotection data in sheep indicate that CMI responses may also 
play a role in the overall protection process $(11,12)$. This is further supported by our observation that the protection elicited by Bac-NS2 protein was abrogated by depletion of $\mathrm{CD} 8^{+}$ $\mathrm{T}$ cells, indicating that at least in mice these cells mediate protection conferred by Bac-NS2 against recombinant VVNS2. This observation, coupled with the cross-protective data obtained from sheep with core-like particles (VP3 and VP7) (12), suggests that a multicomponent vaccine incorporating the major CTL epitopes from these proteins, VP3, VP7, and NS2, could potentially confer effective cross-protection between BTV serotypes if sheep responses mimic those observed in mice. However, in view of the $H$-2 dependence in mice, studies of sheep are required to determine if shared epitopes are selected in different major histocompatibility complex backgrounds.

\section{ACKNOWLEDGMENTS}

We thank P. A. Nuttall, S. Butcher, and E. A. Gould for critically reading the manuscript.

\section{REFERENCES}

1. Bachmann, M. E., T. M. Kündig, G. Freer, C. Y. Kang, D. H. L. Bishop, H. Hengartner, and R. M. Zinkérnagel. 1994. Induction of protective cytotoxic T cells with viral proteins. Eur. J. Immunol. 24:2128-2236.

2. Binder, D., and T. M. Kündig. 1991. Antiviral protection by $\mathrm{CD}^{+} \mathrm{T}$ cells correlating with cytotoxic activity in vitro are more efficient in antivaccinia virus protection than CD4-dependent interleukins. J. Immunol. 146:43014307.

3. French, T. J., J. J. A. Marshall, and P. Roy. 1990. Assembly of doubleshelled, virus-like particles of bluetongue virus by the simultaneous expression of four structural proteins. J. Virol. 64:5695-5700.

4. Gard, G. P., and L. F. Melville. 1992. Results of a decade's monitoring for orbiviruses in sentinal cattle pastured in an area of regular arbovirus activity in Northern Australia, p. 85-89. In T. E. Walton and B. I. Osburn (ed.), Bluetongue, African horsesickness, and related orbiviruses: proceedings of the 2nd International Symposium. CRC Press, Inc., Boca Raton, Fla.

5. Gibbs, E. P. J. 1993. Control of viral diseases of sheep and goats: conventional and novel vaccines, p. 182-199. In R. Pandey, S. Höglund, and G. Prasad (ed.), Veterinary vaccines. Springer-Verlag, New York, N.Y.

6. Hany, M., S. Oehen, M. Schulz, H. Hengartner, M. Mackett, D. H. L. Bishop, H. Overton, and R. M. Zinkernagel. 1989. Anti-viral protection and prevention of lymphocytic choriomeningitis or of the local footpad swelling reaction in mice by immunization with vaccinia-recombinant virus expressing LCMV-WE nucleoprotein or glycoprotein. Eur. J. Immunol. 19:417-424.

7. Jones, L. D., T. Chuma, R. Hails, T. Williams, and P. Roy. 1996. The non-structural proteins of bluetongue virus are a dominant source of cytotoxic T cell peptide determinants. J. Gen. Virol. 77:997-1003.

8. Lawson, C. M., J. R. Bennink, N. P. Resifo, J. W. Yewdell, and B. R. Murphy. 1994. Primary pulmonary cytotoxic T lymphocytes induced by immunization with a vaccinia virus recombinant expressing influenza A virus nucleoprotein peptide do not protect mice against challenge. J. Virol. 68:3505-3511.

9. Offit, P. A., D. B. Boyle, G. W. Both, N. L. Hill, Y. M. Svoboda, S. L Cunningham, R. J. Jenkins, and M. A. McCrae. 1991. Outer capsid glycoprotein VP7 is recognized by cross-reactive rotavirus-specific, cytotoxic T lymphocytes. Virology 184:563-568.

10. Roy, P. 1990. Use of baculovirus expression vectors: development of diagnostic reagents, vaccines and morphological counterparts of blue tongue virus. FEMS Microbiol. Immunol. 64:223-234.

11. Roy, P., D. H. L. Bishop, H. Leblois, and B. J. Erasmus. 1994. Long-lasting protection of sheep against bluetongue challenge after vaccination with virus-like particles: evidence for homologous and partial heterologous protection. Vaccine 12:805-811.

12. Roy, P., J. Callis, and B. J. Erasmus. 1994. Protection of sheep against bluetongue disease after vaccination with core-like and virus-like particles: evidence for homologous and partial heterologous protection, p. 88-97. In Proceedings of the 27th Annual Meeting of the U.S. Health Association.

13. Roy, P., J. J. A. Marshall, and T. J. French. 1990. Structure of the bluetongue virus genome and its encoded proteins, p. 43-88. In P. Roy and B. Gorman (ed.), Current topics in microbiology and immunology: bluetongue viruses. Springer-Verlag, New York, N.Y.

14. Stott, J. L., B. I. Osburn, R. Bushnell, E. C. Loomis, and K. R. E. Squire. 1985. Epizootiological study of bluetongue virus infection in California livestock: an overview. Prog. Clin. Biol. Res. 178:571-582.

15. Tewari, D., J. M. Whalley, D. N. Love, and H. J. Field. 1994. Characterization of immune responses to baculovirus-expressed equine herpesvirus type 1 glycoproteins D and H in a murine model. J. Gen. Virol. 75:1735-1741. 